\title{
Errata: Waveguide evanescent field fluorescence microscopy: high contrast imaging of a domain forming mixed lipid Langmuir-Blodgett monolayer mimicking lung surfactant
}

Abdollah Hassanzadeh and Silvia Mittler

University of Western Ontario, Department of Physics and Astronomy, London, Ontario, N6A 3K7 Canada

[DOI: 10.1117/1.3593467]

This article [J. Biomed. Opt. 16, 046022 (2011)] was originally published on 29 Apr. 2011 with an error in the author affiliations. A footnote has been added to indicate that the author Abdollah Hassanzadeh is currently affiliated with the University of Kurdistan, Department of Physics, Sanandej, Kurdistan, Iran.

The article was corrected online on 6 May 2011. The article appears correctly in print. 